\title{
Designing a road network for hazardous materials shipments
}

\author{
Erhan Erkut ${ }^{\mathrm{a}, *}$, Osman Alp ${ }^{\mathrm{b}}$ \\ ${ }^{a}$ University of Alberta, School of Business, Edmonton, Alta., Canada T6G 2R6 \\ ${ }^{\mathrm{b}}$ Department of Industrial Engineering, Bilkent University, Ankara, Turkey
}

Available online 15 July 2005

\begin{abstract}
We consider the problem of designating hazardous materials routes in and through a major population center. Initially, we restrict our attention to a minimally connected network (a tree) where we can predict accurately the flows on the network. We formulate the tree design problem as an integer programming problem with an objective of minimizing the total transport risk. Such design problems of moderate size can be solved using commercial solvers. We then develop a simple construction heuristic to expand the solution of the tree design problem by adding road segments. Such additions provide carriers with routing choices, which usually increase risks but reduce costs. The heuristic adds paths incrementally, which allows local authorities to trade off risk and cost. We use the road network of the city of Ravenna, Italy, to demonstrate the solution of our integer programming model and our path-addition heuristic.
\end{abstract}

(c) 2005 Elsevier Ltd. All rights reserved.

\section{Introduction}

One of the costs imposed on society by an industrial lifestyle choice is the necessary coexistence with massive amounts of hazardous materials (hazmats) in our daily lives. Large quantities of hazmats are shipped on trucks-one in five trucks on North American highways carries hazmats, and there are 800,000 road shipments per day [1]. While the safety record of the industry is quite good, accidents do happen, and sometimes the consequences are severe. In 1998, 13 fatalities occurred and 432 accidents were classified as "serious incident" in the United States [2]. The public is concerned about the potential for catastrophes. Authorities at different levels take measures to reduce and mitigate the risks associated

\footnotetext{
* Corresponding author.

E-mail address: erhan.erkut@ualberta.ca (E. Erkut).
} 
with the transportation of hazmats. One tool available to local authorities is the restriction of hazmats transport to certain roads. For example, the local authority bylaws section of the Alberta Dangerous Goods Transportation and Handling Act [3] reads:

17(1) Subject to the regulations, a local authority within the meaning of the Municipal Government Act or a Métis settlement may make bylaws with respect to highways under its direction, control and management

(a) designating the route and time of travel of vehicles transporting dangerous goods,

(b) prohibiting the carriage of dangerous goods on those highways specified in the bylaw, and

(c) specifying restrictions or conditions to ensure the safe transportation in or by a means of transport, safe storage and controls necessary for public safety.

This paper develops a methodology that can be used to identify hazmats routes in (and through) a major population center.

While transport risks can be reduced by limiting access on city roads to hazmat vehicles, municipalities must also provide adequate access to local businesses and organizations that may be origins or destinations of these shipments. Carriers will incur increased costs due to limited routing available, and such costs will be passed on to consumers. Hence, the problem at hand is one of trading off economic costs and public risks.

Consider the road network in and around a major population center. Suppose there are a number of origins and destinations for hazmat shipments in this city. There are two relevant actors that determine the hazmat flows in the city: the local authority and the hazmat carriers. The local authority restricts hazmat shipments to a subset of the roads in the city, identified as the "hazmat network," and the carriers select routes between their origins and destinations on this hazmat network. While the local authority is primarily interested in minimizing public risk, the carriers are concerned about minimizing transport costs. It is fair to assume that the local authority will design a hazmat network with risk minimization in mind, and that the carriers will select the most economic paths on this network.

Considering the rational response of the carriers, how should the local authority design the hazmat network so as to minimize risk? Given appropriate data and a selected method to quantify risk (see Erkut and Verter [4] for a discussion of various methods), it is simple to find a minimum risk route for each origin-destination (OD) pair. However, declaring the union of these routes as the hazmat network may not minimize risk in practice, since carriers are not obliged to take minimum risk routes. While the local authority is able to designate the hazmat network, it cannot force specific routes on individual shipments. The carriers are required to stay on the hazmat network, but they are free to select their routes, and they may not select a minimum risk route in the presence of multiple routes. This choice complicates the hazmat network design problem, since the local authority must consider the decisions of the carriers in response to the hazmat network design. It is possible to consider these decisions, as we discuss in Section 3 , but the resulting optimization problem is difficult to solve.

One way to simplify the hazmat network design problem is to provide no alternatives for the shipments, in other words, to design a hazmat network such that there is only one route between any given origin and destination. The result would be a tree network, a network with no cycles. This network is the sparsest possible that is still connected. In addition to giving the local authority full control over the routing of hazmat shipments, there are other benefits of a sparse hazmat network. If the local authority is planning to improve the conditions of the roads on the hazmat network to reduce accident probabilities 
(for example, by resurfacing the road, providing turn lanes, wider shoulders, or better lighting) a sparse network minimizes improvement costs. Also, a sparse network may be easier to control and police. Finally, emergency response planning and delivery can be easier on a sparse network. While there are some benefits of a tree design, it may not be acceptable as a city hazmat network, since it may impose excessive transport costs on the carriers. Whether or not a tree is a viable design will depend on the street network and the hazmat flows.

In this paper we formulate the hazmat network design problem as a tree selection problem and discuss how this sparse network can be expanded to offer some choice to carriers. We believe that this alternative may be viable for designing a hazmat network. In the next section we discuss the relevant literature. In Section 3 we provide the general formulation of the hazmat network design problem and the formulation for the tree network design problem. We discuss the solution method for the tree network design problem in Section 4. In Section 5 we describe a method to expand the optimal tree that results in a decrease of transport costs (usually at the expense of increased risk). Section 6 contains a numerical experiment and the results of our computational experience with the tree design model and the algorithm. In Section 7 we introduce the bicriterion version of the tree network design problem and the tree expansion algorithm. Section 8 concludes the paper.

\section{Literature}

Two main streams of research are relevant to this paper: hazmat transportation and network design. Both streams are rich in papers, and we will limit our review to papers that are directly relevant. IRR [5] and CCPS [6] offer excellent general resources for hazmat transport risk assessment. Erkut et al. [7] provide a review of the operations research literature in the area of hazmat transportation. Magnanti and Wong [8] review network design and transportation planning models. Yang and Bell [9] provide a more recent review of network design problems for road transportation.

The tree network design problem we consider is similar to the "optimum communication spanning tree problem" (OCST), where the objective is to minimize the weighted sum of the lengths of the paths between each pair of nodes on a tree. There is considerable literature on this problem that was first introduced by $\mathrm{Hu}$ [10]. Hu studied two special cases of this problem: an optimum requirements spanning tree (all distances between nodes are unity) and an optimum distance spanning tree (all flow requirements are unity). $\mathrm{Hu}[10]$ presents an $O\left(n^{4}\right)$ algorithm for the former case and develops a number of sufficiency conditions for the latter case. Fischetti et al. [11] devise a branch-and-bound-based exact algorithm for the optimum distance spanning tree problem. Ahuja and Murty [12] propose an exact and a heuristic solution algorithm for OCST. The exact algorithm utilizes the lower plane concept developed by the authors to generate tight lower bounds, and these bounds are used in a branch-and-bound algorithm. The authors also propose a heuristic algorithm that consists of two phases. In the first phase, a construction heuristic is implemented to establish a tree. The second phase tries to improve upon this initial solution. Palmer and Kershenbaum [13] and Li and Bouchebaba [14] propose genetic algorithms for OCST. Wu et al. [15] propose polynomial time 1.577- and 2-approximation algorithms for slightly different versions of OCST. In the first version, the requirements of the OD pairs are assumed to be the product of their weights, and in the second version, the requirements are assumed to be equal to the sum of their weights. Finally, Peleg and Reshef [16] propose a logarithmic approximation for OCST based on the concept of minimum average stretch spanning trees for weighted connected multigraphs. 
We are aware of only one paper that lies at the intersection of the hazmat transportation and network design literatures. Kara and Verter [17] formulate the design problem as a bi-level mathematical programming model. They assume the carriers will always use the shortest paths on the hazmat network. The objective for the authority is to select the minimum risk network, taking into account the cost-minimizing behavior of the carriers. This model is more general than ours, since it does not limit the hazmat network to a tree. However, their resulting mathematical model is quite large and is not computationally feasible for large networks. In contrast, our model is smaller and can be solved optimally for realistic-sized networks. In addition, there exist heuristics [18] to solve larger instances of the tree network design problem. Perhaps more importantly, the bi-level model is a single objective model that minimizes risk. In contrast, our approach provides more freedom to the decision maker by allowing for cost-risk tradeoffs, as the minimum risk tree is expanded by link additions.

\section{Formulations}

First, we define our notation and provide a formal definition of the general bi-level hazmat network design problem. Then we offer a formulation for OCST and the hazmat tree design problem.

Let $N$ be the set of nodes on a city road network and $E$ be the set of undirected edges $(i, j)$ that connect these nodes. Without loss of generality, let $i<j$ for the edges in set $E$. Let $C$ be the set of pairs of nodes $(u, v)$ such that $u<v$, where there exists a positive shipment between nodes $u$ (origin) and $v$ (destination). Moreover, let

$n \quad$ number of nodes in $N$,

A $\quad\{(i, j),(j, i):(i, j) \in E\}$,

$s_{u v} \quad$ number of shipments between nodes $u$ and $v$ where $(u, v) \in C$,

$r_{i j} \quad$ risk per shipment on $\operatorname{arc}(i, j) \in A$,

$l_{i j} \quad$ length of $\operatorname{arc}(i, j) \in A$.

How risk is quantified is up to the decision maker. If one uses the traditional definition of risk, it would be equal to the product of the incident probability on $\operatorname{arc}(i, j)$ and the consequence (which could be equal to the number of persons impacted in case of an incident). For our purposes, we assume that the per-shipment risk is known on each arc and is independent of the direction of the shipment. The formulation provided is for a single hazmat commodity. However, it is straightforward to generalize it to the case of multiple hazmats by introducing a commodity index. In this case, we would need to compute the risk coefficients for each commodity.

The decision variables are

$$
\begin{aligned}
& X_{i j}= \begin{cases}1 & \text { if } \operatorname{arc}(i, j) \text { is on the hazmat network, } \\
0 & \text { otherwise, }\end{cases} \\
& Z_{i j}^{u v}= \begin{cases}1 & \text { if }(i, j) \text { is on the path connecting nodes } u \text { and } v, \\
0 & \text { otherwise. }\end{cases}
\end{aligned}
$$

Using our notation, the bi-level hazmat network design problem posed by Kara and Verter [17] can be stated as follows:

$$
\min _{X_{i j} \in\{0,1\}} \sum_{(i, j) \in A} \sum_{(u, v) \in C} s_{u v} r_{i j} Z_{i j}^{u v}
$$


where $Z_{i j}^{u v}$ solves

$$
\begin{aligned}
& \min \sum_{(u, v) \in C} \sum_{(i, j) \in A} s_{u v} l_{i j} Z_{i j}^{u v} \\
& \text { s.t. } \quad \sum_{(i, k) \in A} Z_{i k}^{u v}-\sum_{(k, i) \in A} Z_{k i}^{u v}=\left\{\begin{array}{ll}
+1, & i=u, \\
-1, & i=v \\
0, & \text { o.w. },
\end{array} \quad \forall i \in N,(u, v) \in C,\right. \\
& Z_{i j}^{u v} \leqslant X_{i j} \quad \forall(i, j) \in A,(u, v) \in C, \\
& Z_{i j}^{u v} \in\{0,1\} .
\end{aligned}
$$

The inner decision problem takes the decision by the local authorities (the values of the $X$-variables) as given and solves a cost minimization problem over the $Z$-variables. This part represents the cost minimization by the carriers. The outer decision problem deals with the selection of the hazmat links (i.e. the design of the hazmat network). Constraint (1) is the flow conservation constraint, and constraint (2) limits the use of the network links to those selected as hazmat links.

It is possible to solve this problem by conducting a search over the design variables and solving a series of linear programs for each selection of design variables. Alternately, the problem can be converted to a single level optimization problem by writing out the Karush-Kuhn-Tucker optimality conditions (constraints 3-6) using additional variables $(w, v, \lambda)$ and a large number $R$.

$$
\begin{aligned}
& \min \sum_{(i, j) \in A} \sum_{(u, v) \in C} s_{u v} r_{i j} Z_{i j}^{u v} \\
& \text { s.t. } \quad \sum_{(i, k) \in C} Z_{i k}^{u v}-\sum_{(k, i) \in C} Z_{k i}^{u v}=\left\{\begin{array}{ll}
+1, & i=u, \\
-1, & i=v \\
0, & \text { o.w. }
\end{array} \quad \forall i \in N, \quad(u, v) \in C,\right. \\
& Z_{i j}^{u v} \leqslant X_{i j} \quad \forall(i, j) \in A,(u, v) \in C, \\
& s_{u v} l_{i j}-w_{i}^{u v}+w_{j}^{u v}-v_{i j}^{u v}+\lambda_{i j}^{u v}=0 \quad \forall(i, j) \in A,(u, v) \in C, \\
& v_{i j}^{u v} \leqslant R\left(1-Z_{i j}^{u v}\right) \quad \forall(i, j) \in A,(u, v) \in C, \\
& \lambda_{i j}^{u v \leqslant R}\left(1-\left(X_{i j}-Z_{i j}^{u v}\right)\right) \quad \forall(i, j) \in A,(u, v) \in C, \\
& v_{i j}^{u v} \geqslant 0, \quad \lambda_{i j}^{u v} \geqslant 0, \quad w_{i}^{u v} \text { free, } \quad Z_{i j}^{u v} \in\{0,1\}, \quad X_{i j} \in\{0,1\} .
\end{aligned}
$$

This is a mixed integer programming problem, which can be solved using an off-the-shelf solver. However, the problem size may make it difficult to solve realistic versions of the problem. For a problem with $n$ nodes, $k$ OD pairs, and $m$ arcs, the formulation contains $n k+4 m k$ constraints, $m(k+1)$ binary variables, and $2 k(m+n)$ non-negative continuous variables. For example, with 100 nodes, 30 OD pairs, and 200 arcs, the mathematical program has 27,000 constraints, 6,200 binary variables, and 18,000 continuous variables. In contrast, restricting the solution to a tree network, as we consider next, results in a more compact mathematical model.

We now turn our attention to the tree network design problems. First, we provide the formulation for OCST. This formulation is later extended to minimum risk hazmat tree (MRHT). 
(OCST):

$$
\begin{aligned}
& \min \sum_{(u, v) \in C} \sum_{(i, j) \in A} s_{u v} r_{i j} Z_{i j}^{u v} \\
& \quad \sum_{(i, j) \in A} Z_{i j}^{u v}-\sum_{(j, i) \in A} Z_{j i}^{u v}= \begin{cases}1 & \forall i \in N,(u, v) \in C, i=u, \\
0 & \forall i \in N,(u, v) \in C, i \neq u, v,\end{cases} \\
& Z_{i j}^{u v}+Z_{j i}^{u v} \leqslant X_{i j} \quad \forall(i, j) \in E,(u, v) \in C, \\
& \sum_{(i, j) \in E} X_{i j}=n-1, \\
& Z_{i j}^{u v} \in\{0,1\} \quad \forall(i, j) \in A,(u, v) \in C, \\
& X_{i j} \in\{0,1\} \quad \forall(i, j) \in E .
\end{aligned}
$$

Constraint set (7) provides the flow conservation constraints for each shipment. Constraint set (8) guarantees that if there is a shipment on an arc, then it must be part of the hazmat tree. Constraint (9) requires the selection of exactly $n-1$ arcs, which guarantees the construction of a tree network. Constraint sets (10) and (11) declare the decision variables as binary.

OCST is a special case of the hazmat tree design problem, since it assumes that each network node is either an origin or a destination. However, many nodes of the transport network will be transhipment nodes (i.e. neither supply nor demand points), and the set of origins and destinations will be a (small) subset of the node set. What we want is not an optimum communication spanning tree, but an optimum communication Steiner tree. The Steiner tree would span all origins and destinations and a subset of the remaining nodes. While there is considerable literature on OCST, to the extent of our knowledge, the optimum communication Steiner tree problem has received no attention.

The formulation of the optimum communication Steiner tree is presented below; we call this the MRHT. The nodes are classified into two sets: mandatory and non-mandatory nodes. Origins and destinations are mandatory nodes, and the rest are non-mandatory. Let $S$ and $T(S \cup T=N, S \cap T=\emptyset)$ be the set of mandatory and non-mandatory nodes, respectively. Define $Z$ and $X$ decision variables similar to the decision variables in the previous formulation (but define $X$ in both directions). One of the mandatory nodes should be designated as the root node with node number one. We assume that there is non-zero traffic between the sets of nodes $U$ and $N \backslash U$, for all $U \subset N$. This assumption does not cause a loss of generality, since otherwise, we can solve two separate problems for the sets of nodes $U$ and $N \backslash U$. The formulation of MRHT follows:

(MRHT):

$$
\begin{gathered}
\min . \sum_{(u, v) \in C} \sum_{(i, j) \in A} s_{u v} r_{i j} Z_{i j}^{u v} \\
\sum_{(i, j) \in A} Z_{i j}^{u v}-\sum_{(j, i) \in A} Z_{j i}^{u v}= \begin{cases}1 & \forall i \in N,(u, v) \in C, \quad i=u, \\
0 & \forall i \in N, \quad(u, v) \in C, i \neq u, v,\end{cases} \\
Z_{i j}^{u v}+Z_{j i}^{u v} \leqslant X_{i j}+X_{j i} \quad \forall(i, j) \in E: i<j, \quad(u, v) \in C,
\end{gathered}
$$




$$
\begin{aligned}
& \sum_{j \in N} X_{i j}=0 \quad \text { for } i=1, \\
& \sum_{j \in N} X_{i j}=1 \quad \text { for } i \neq 1 \text { and } i \in S, \\
& \sum_{j \in N} X_{i j} \leqslant 1 \quad \text { for } i \in T, \\
& Z_{i j}^{u v} \in\{0,1\} \quad \forall(i, j) \in A,(u, v) \in C, \\
& X_{i j} \in\{0,1\} \quad \forall(i, j) \in A .
\end{aligned}
$$

Constraints (14)-(16) are the structural constraints for the Steiner tree. Each mandatory node (except the root node) should have only one outgoing arc. The root should have only incoming arcs. The Steiner nodes, on the other hand, may have at most one outgoing arc. If a particular Steiner node is on the tree, then it should have one outgoing arc, otherwise no outgoing and incoming arcs should exist for that node. Constraint set (12) maintains the flow on the tree. Constraint set (13) relates the flow variables to structural variables.

\section{Solving the problem}

Despite the restriction of the solution to a (Steiner) tree network, this design problem is difficult to solve. It is well known that the Steiner tree problem is NP-hard [19]. Yet it is well studied, and there exist for it a number of effective heuristics (for example, the tabu search by Gendreau et al. [20]) and efficient optimal algorithms (such as Polzin and Daneshmand [21]), and large instances can be solved to optimality. However, we cannot use a Steiner tree algorithm for MRHT. The total risk imposed on an arc depends on the flow on that arc, which in turn depends on the topology of the tree selected. Hence, it is not possible to compute the total risk on an arc in advance, which rules out the use of Steiner tree algorithms.

For a design problem with $n$ nodes (of which $s$ are mandatory), $m$ arcs and $k$ OD pairs, MRHT has $(m k+m)$ variables and $(n k+k m / 2+s)$ constraints. For example, with 100 nodes (of which 20 are mandatory), 30 OD pairs, and $200 \operatorname{arcs}$ (namely $n=100, s=20, m=200$, and $k=30$ ), the design problem will have 6,200 variables and 6,020 constraints. Table 1 compares the problem sizes for the bi-level model and MRHT as a function of problem parameters. Note that while the number of binary variables is the same, the bi-level model has many continuous variables that are missing from MRHT. Furthermore, the number of constraints of the bi-level model grows much more rapidly. For the computational experience described in Section 6, we solved the MRHT with 105 nodes, 135 arcs, 12 mandatory nodes, and 35 OD

Table 1

A comparison of the problem size of the bi-level model and MRHT as a function of problem parameters

\begin{tabular}{llll}
\hline & Constraints & Binary var. & Continuous var. \\
\hline Bi-level & $n k+4 m k$ & $m(k+1)$ & $2 k(m+n)$ \\
MRHT & $n k+m k / 2+s$ & $m(k+1)$ & 0 \\
\hline
\end{tabular}

$n$, number of nodes; $m$, number of arcs, $k$, number of OD pairs and $s$, number of mandatory nodes $(\leqslant 2 k)$. 
pairs using CPLEX 8.0 in $15 \mathrm{~s}$ on a $750 \mathrm{MHz}$ Sun Blade 1000. In contrast, three days of CPU time on the same problem failed to generate an optimal solution to the bi-level model.

For larger instances of MRHT, one may have to resort to a heuristic algorithm. In Erkut and Alp [18] we describe several construction and improvement heuristics for MRHT and compare them empirically. The construction heuristics add arcs or paths until a tree network is created, and the improvement heuristics consider exchanges of arcs or paths in the solution. These heuristics are very fast and are able to find excellent solutions.

\section{Expanding the tree network}

While we are able to solve MRHT (either optimally or approximately), a tree design for a hazmat transport network may not be economically acceptable, due to the imposition of circuitous and excessively long routes on the carriers. If one restricts travel to a tree network, a short trip on the general network can be turned into a long trip on the tree. For example, consider a simple network that consists of the edges of the unit square. A tree can be obtained by deleting any one of the edges. Assume that the nodes incident to the deleted edge are the origin and destination for one shipment. The path length for this shipment goes from 1 (minimum possible) to 3 (maximum possible for acyclic paths) upon the deletion of the edge, which implies a tripling of the transport cost. Note that this factor of increase can be made arbitrarily large by extending the network to a mesh network. While this example is pathological, it makes the point effectively.

It is reasonable to assume that the carriers would be interested in minimizing transport costs. Some increase in their transport costs might be tolerated for purposes of risk minimization and emergency response. However, the tree design is likely to result in considerable increases in transport costs. Hence, it may be desirable to extend the tree network generated by MRHT by adding some edges. The addition of edges may result in increases to the total risk, since on a cyclic network the carriers would select cost-minimizing paths that may not be the same as risk-minimizing paths. The local authority may be interested in expanding the tree network by selecting an upper bound on the maximum risk. In this section, our goal is to expand the network by adding selected paths to the tree, so as to keep the risk increase to a minimum. The paths we consider for insertion are shortest paths, and this selection guarantees a reduction in transport costs.

Given that MRHT is a restriction of the general hazmat network design problem where the network topology is not dictated, the solution to MRHT is a feasible (but not necessarily optimal) solution to the general problem. As the following simple example demonstrates, the addition of arcs will not only decrease transport costs, but may also decrease the total risk. Consider an equilateral triangle transport network with nodes $\mathrm{A}, \mathrm{B}$, and $\mathrm{C}$, and the following simple shipment pattern: $\mathrm{AB}, \mathrm{BC}$, and $\mathrm{CA}$. Assume the materials shipped are different. Suppose all shipment volumes are equal to one unit and that all unit risks on arcs are equal to one. For this problem, deleting any one of the arcs provides an optimal solution to MRHT. Suppose we select the solution AB, BC. The shipment from C to A must travel via node B, and the total risk in the system is 4 . If we add the arc CA to the tree, then the total risk goes down to 3. As this example demonstrates, adding arcs and paths to the solution of MRHT will not only reduce transport costs, but it may also reduce the total risk.

We propose a greedy algorithm that adds paths to the current network. At each iteration, we consider the shortest paths between all OD pairs on the network, and we insert the path(s) that results in the smallest 
increase in the total risk. In addition to a network that is denser than a tree, such a greedy insertion algorithm produces a risk-cost tradeoff curve that could be useful for a decision maker. We now describe the algorithm formally.

A greedy insertion algorithm can insert one or more paths per iteration. We summarize $\operatorname{Greed}(q)$ below, which would insert $q$ paths per iteration. We implement this algorithm for $q=1$ and 2 .

$\operatorname{Greedy}(q)$ :

1. Let set $\Pi=\left\{\left(u_{i}, u_{j}\right): i<j ; i, j \in\{1,2, \ldots, K\}\right\}$ where $\left\{u_{1}, u_{2}, \ldots, u_{K}\right\}$ is the set of nodes of $H$.

2. Find the shortest path between $u_{i}$ and $u_{j}$ for all node pairs.

3. Let $V$ be the set of all subsets of $\Pi$ with exactly $q$ elements.

4. Find the element of $V$ that results in the minimum total risk $T R$ when inserted to $H$.

5. Update $H$ by inserting the selected edges.

Main algorithm:

1. Solve MRHT for the given problem.

2. Let $H$ be the resulting tree network.

3. Find total cost $T C$ and total risk $T R$ for the current $H$.

4. Implement Greedy $(q)$ for the selected value of $q$ repeatedly until no further reduction in cost is possible.

5. Among the solutions generated, select an efficient subset with respect to the cost and risk criteria for presentation to the decision maker.

This algorithm considers both cost and risk without combining the two into a single selection rule. Only shortest paths are considered for insertion. Hence, when $q=1$, for a given pair of nodes, the inserted path is the one that results in the largest possible reduction in transport cost for all shipments between the two nodes. Among such paths (which can be considered locally optimal with respect to the cost criterion), we select the one that results in the smallest increase in the total risk. Alternately, we could find minimum risk paths for all pairs of nodes and then select the one that results in the largest reduction in cost, as a variant of our algorithm.

\section{Computational experiment}

We tested our algorithm on the network for the city of Ravenna, Italy. This network has 105 nodes and 134 edges. We have origin-destination data on five different hazmats on this network (LPG, methanol, gasoline, gas oil, and chlorine). We simplified the problem by combining these five commodities into a single commodity. There are 12 nodes with a transportation requirement. These nodes constitute the set of mandatory nodes in the MRHT formulation. Transport risk is computed by multiplying the frequency of release of the hazmats in case of an accident with a population figure. The problem data allow us to model population using the following components:

(i) population density on the arcs (persons/meter),

(ii) population density in the proximity of arcs (within a given bandwidth),

(iii) locations of and population density at population concentration points (schools, hospitals, theaters, commercial centers, etc.). 


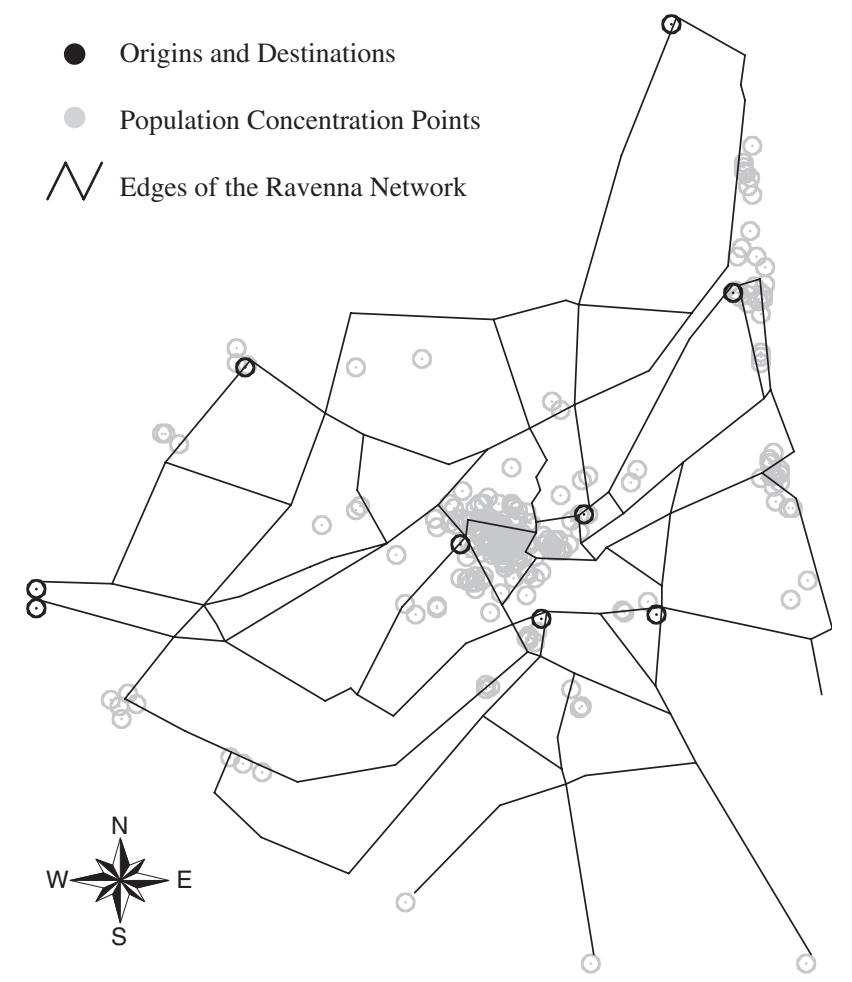

Fig. 1. The road network of the city of Ravenna, Italy. The black dots indicate the origins and destinations of hazmat shipments. The gray dots represent the locations of population concentration points, such as schools and theaters.

While it is possible to produce a comprehensive risk measure by combining these three sources of data, below we summarize the results we get when using population concentration points within $500 \mathrm{~m}$ of an arc. There are 248 population concentration points, and most are clustered in the center of the city. Fig. 1 shows the road network, the OD nodes (black dots), and the population concentration points in the city (gray dots). On this network, the minimum total shipment cost is 2,509,040 truck-kilometres.

Fig. 2 displays the optimal solution to MRHT on this network. The total transport distance is 5,680,660 truck-kilometres. This value is equal to 2.26 times the minimum possible distance on the full network. The total risk associated with the MRHT solution is 1254.81 (this is the expected number of impacted persons). This number is slightly less than $6 \%$ of the risk associated with the case where the carriers are allowed to choose minimum cost paths from the entire network. Hence, restricting the full network to the MRHT reduces the risk by over $94 \%$ but increases the cost by $126 \%$. This result suggests that a drastic risk reduction (an order of magnitude) is possible by limiting the hazmat transport network to a tree, if one is willing to double the transport cost.

Using Greedy(1), the path addition algorithm adds a total of 23 paths to the initial tree to attain the lowest possible total cost. The addition of these paths creates a network that consists of the union of all OD shortest paths. Some of these additions change the cost and risk measures very little. For example, 


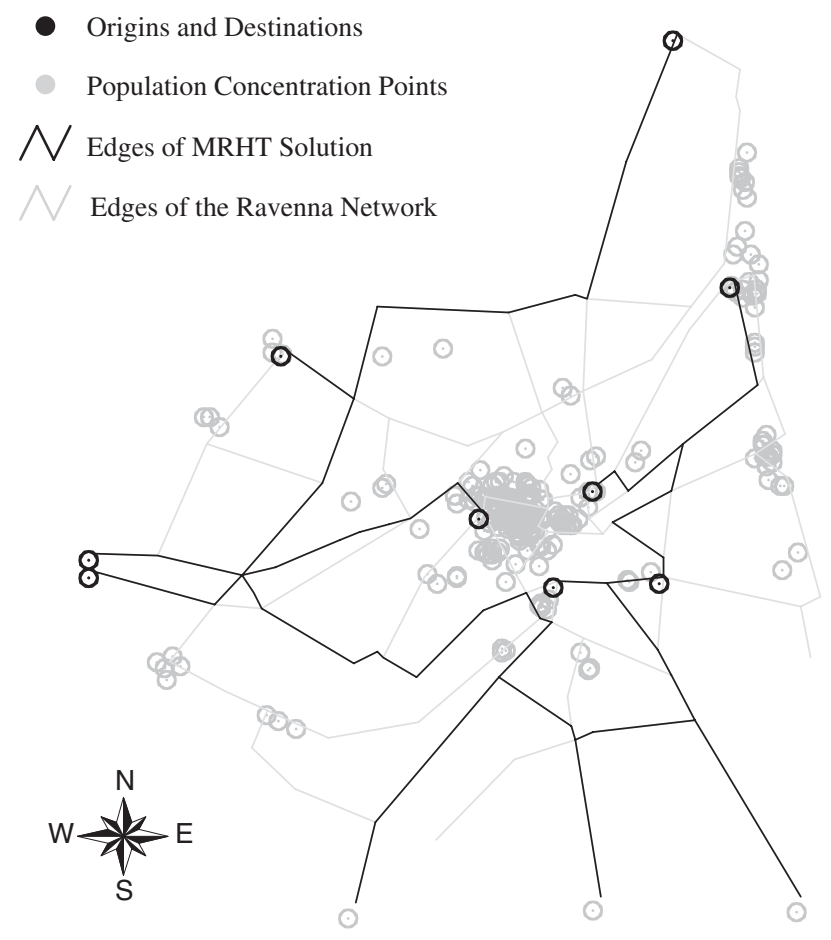

Fig. 2. The solution of MRHT on the Ravenna network.

adding the first path results in no change to the risk measure and a $0.26 \%$ reduction in the cost. This path consists of a single shortcut arc. The rerouting of shipments to this arc imposes the same risk as on MRHT but reduces the cost slightly. This solution suggests that there exist multiple optima for the MRHT problem (Note that from a bicriterion optimization perspective, MRHT is dominated by the new network, which consists of the union of MRHT with the added shortcut arc.).

The second added path increases risk by $0.04 \%$ and reduces cost by $0.002 \%$. The third one increases risk by $0.16 \%$ and reduces cost by $0.02 \%$. Fig. 3 displays the solution after the addition of these first three paths. In comparison to MRHT, this solution is associated with $0.20 \%$ more risk and $0.28 \%$ less cost. Fig. 4 displays the solution after the addition of 10 paths. In comparison to MRHT, this solution is associated with $11.7 \%$ more risk and $24.5 \%$ less cost. When compared to the case where carriers are allowed to choose paths from the full network, the risk associated with this solution is only $6.3 \%$ of the risk on the full network, and the cost is $71 \%$ higher than the risk on the full network.

If one adds 22 paths (i.e. all but the last one), the solution generated is only $5.7 \%$ more costly than the one on the full network, but is associated with only $36.5 \%$ of the risk of the full network. In this instance, the closure of a few arcs results in a drastic reduction in risk at a relatively minor increase in cost.

We have also experimented with Greedy(2). This algorithm takes about five times as long as Greedy(1) (50 s vs. 10 s on a $750 \mathrm{MHz}$ Sun Blade 1000). It stops after 13 iterations, inserting 26 paths. Some of the iterations (6 of 13) duplicate a solution found by Greedy(1), while others generate a different efficient solution. As one would expect, some of the solutions generated by Greedy(2) dominate those generated by Greedy(1). This is the case with three of the 23 solutions produced by Greedy(1). The solutions generated 
- Origins and Destinations

Population Concentration Points

$\bigwedge$ Edges of MRHT Solution

Paths Added

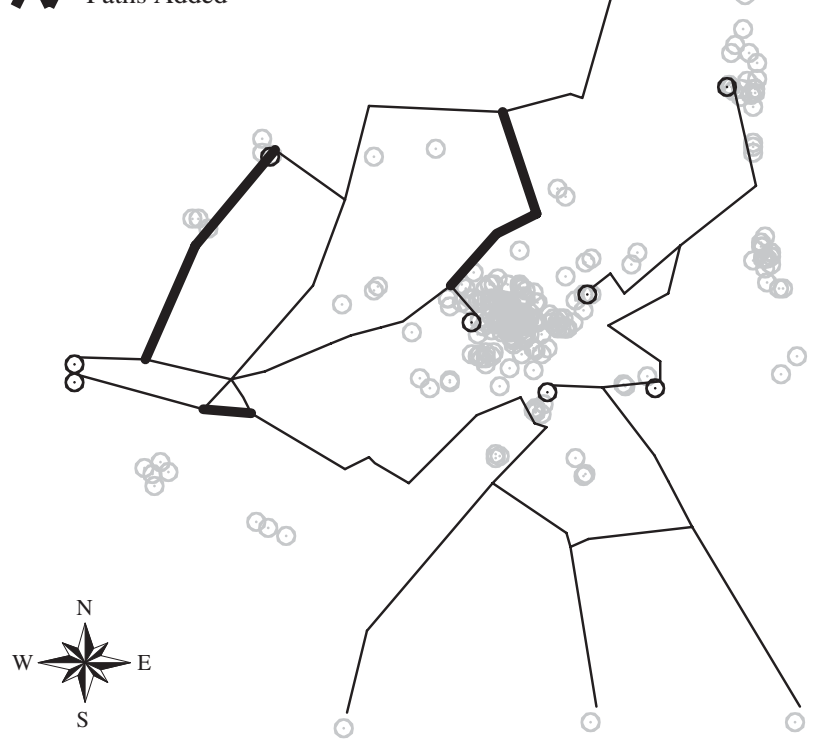

Fig. 3. The solutions generated after adding three paths to MRHT.

- Origins and Destinations

Population Concentration Points

$\bigwedge$ Edges of MRHT Solution

Paths Added

$w \rightarrow \overbrace{s}^{N} E$

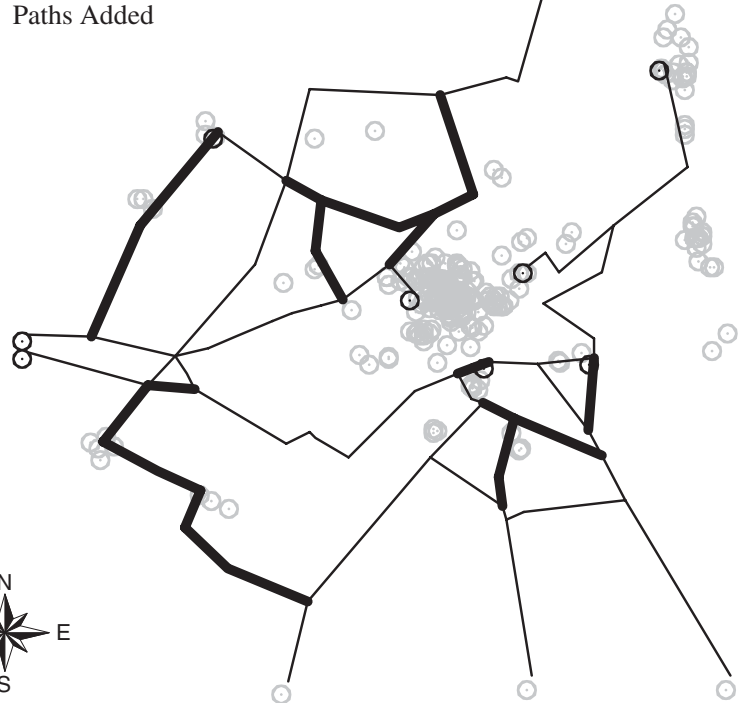

Fig. 4. The solutions generated after adding 10 paths to MRHT. 
Table 2

A comparison of the solutions generated by Greedy(1) and Greedy(2) after adding 18, 20, and 22 paths to the starting tree solution. The Greedy(2) solutions dominate the Greedy(1) solutions in these three cases

\begin{tabular}{|c|c|c|c|c|c|c|}
\hline & \multicolumn{6}{|c|}{ Number of paths added } \\
\hline & \multicolumn{2}{|l|}{18} & \multicolumn{2}{|l|}{20} & \multicolumn{2}{|l|}{22} \\
\hline & $T R$ & $T C$ & $T R$ & $T C$ & $T R$ & $T C$ \\
\hline Greedy(1) & $566,954,000$ & $3,830,170,000$ & $703,058,000$ & $2,861,340,000$ & $694,171,000$ & $2,802,370,000$ \\
\hline Greedy(2) & $497,544,000$ & $3,634,580,000$ & $599,247,000$ & $2,808,020,000$ & $659,006,000$ & $2,702,490,000$ \\
\hline Difference & $12 \%$ & $5 \%$ & $15 \%$ & $2 \%$ & $5 \%$ & $4 \%$ \\
\hline
\end{tabular}

at the 9th, 10th, and 11th iterations of Greedy(2) dominate the solutions generated at iterations 18, 20, and 22 of Greedy(1). Table 2 compares these three pairs of solutions. It seems that supplementing Greedy(1) with Greedy(2) adds more information for decision makers.

Of course, all of the figures discussed in this section are dependent on the problem data on hand, but we believe this numerical example demonstrates the potential use of our algorithm in designing a hazmat transport network.

\section{Bi-criterion design problem}

MRHT minimizes risk, and the greedy link addition algorithm selects shortest paths that increase risk as little as possible. It is possible to extend this methodology to account for the bi-criterion nature of the problem. Although MRHT is a single-objective model that minimizes risk, it can be argued that cost minimization is also a first-level design objective, since imposing excessive costs on carriers will have an adverse impact on the economy.

Let $\alpha$ and $\beta$ be two parameters where $0 \leqslant \alpha \leqslant 1$ and $\beta \geqslant 0$. Let $f(T R, T C)=\alpha \beta T R+(1-\alpha) T C$, where $T R$ and $T C$ are the total risk and total transportation costs on a given network, respectively. The parameter $\alpha$ is the weight assigned to the risk objective relative to the cost objective, and $\beta$ is a scale parameter that makes the values of the risk measure comparable to the values of the cost measure. By replacing the objective function of MRHT with this weighted function, we obtain the bi-criterion version of MRHT, which we call MRCHT (minimum risk-cost hazmat tree):

$(\operatorname{MRCHT}(\alpha))$ :

$$
\begin{array}{ll}
\text { Min. } & \alpha \beta \sum_{\substack{(u, v) \in C \\
\text { s.t. }}} \sum_{(i, j) \in A} s_{u v} r_{i j} Z_{i j}^{u v}+(1-\alpha) \sum_{(u, v) \in C} \sum_{(i, j) \in A} s_{u v} l_{i j} Z_{i j}^{u v} \\
& (17) .
\end{array}
$$

We can solve $\operatorname{MRCHT}(\alpha)$ for different values of $\alpha$ and generate several trees with different total risk and cost values. The decision maker can select one of these solutions. Table 3 summarizes different solutions for three different values of $\alpha$ in the Ravenna network for gasoline shipments only, when the risk proxy is estimated by the population concentration points (we used $\beta=100$ to scale risk values in MRCHT $(\alpha)$ ). When $\alpha$ is changed from 1.0 to 0.6 , TR increases by only $2.7 \%$ but TC decreases by almost $30 \%$. 
Table 3

Three different solutions to $\operatorname{MRCHT}(\alpha)$ for the Ravenna network with different weights for risk and cost

\begin{tabular}{llr}
\hline$\alpha$ & $T R$ & $T C$ \\
\hline 1.0 & $39,436,400$ & $2,895,030,000$ \\
0.6 & $40,511,300$ & $2,030,470,000$ \\
0.0 & $1,102,730,000$ & $1,095,480,000$ \\
\hline
\end{tabular}

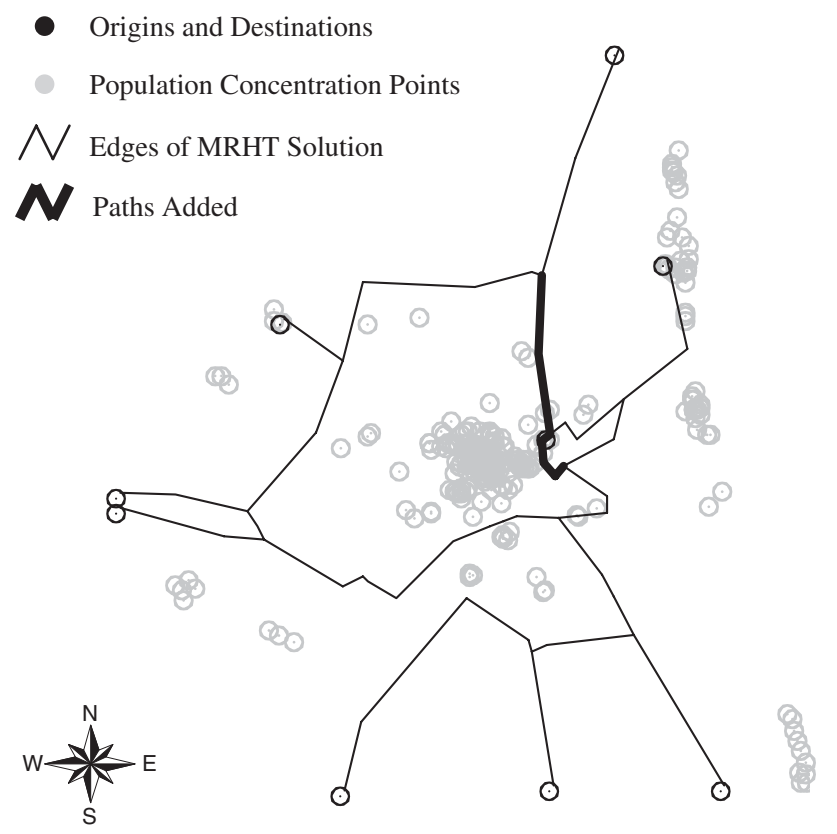

Fig. 5. A solution obtained by implementing Greedy(1,0) starting with MRCHT(0.6).

A similar weighting approach can be used in the greedy addition algorithm. In Step 4, instead of using $T R$ only, we would use a weighted sum of $T R$ and $T C$. Starting from any given tree solution, we can implement $\operatorname{Greed} y(q, \alpha)$ for different values of $\alpha$ and generate several alternatives for the decision maker. To test this methodology, we applied Greedy $(1, \alpha)$ for $\alpha=1.0,0.6,0.0$ to each of the three starting solutions summarized in Table 3 and generated a set of 25 non-dominated solutions. Fig. 5 depicts a solution obtained by implementing Greedy(1,0) starting with MRCHT(0.6). In this solution, the total risk is 5.38 times more than the minimum risk possible, but the total cost is only 1.33 times more than the minimum possible cost. We display a subset of the non-dominated solutions in Fig. 6 (excluding those that are associated with more than eight times the minimum risk found). It may be possible to generate more solutions by using other values of $\alpha$ in solving $\operatorname{MRCHT}(\alpha)$ and/or by applying Greedy $(1, \alpha)$. However, this method does not guarantee the generation of the exact efficient frontier to the bi-criterion problem, since the two-phase method is a heuristic solution procedure for this problem. 


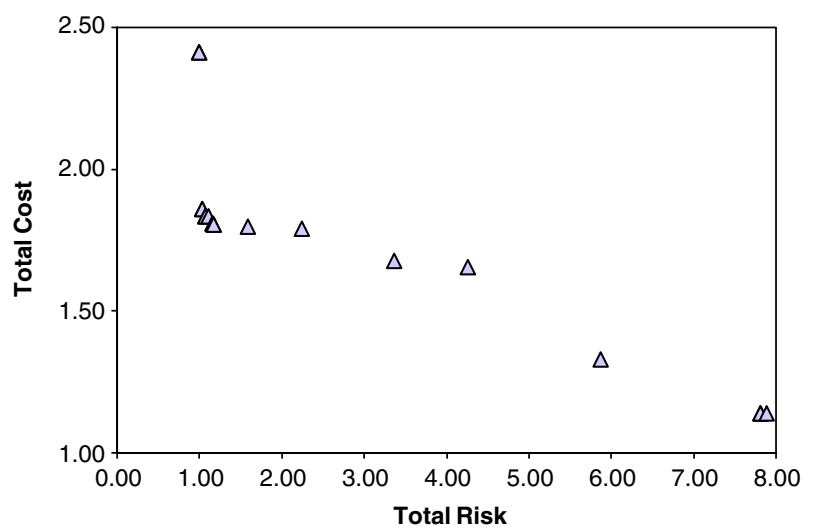

Fig. 6. A subset of the non-dominated solutions generated by solving MRCHT( $\alpha)$ and applying $\operatorname{Greedy}(1, \alpha)$ for $\alpha=1.0,0.6$, and 0.0. The risk and cost values are scaled (The six solutions associated with more than eight times the minimum risk found are not shown.).

\section{Concluding remarks}

In this paper, we are concerned with designing a hazmat transport network by selecting a subset of all links on a road network. We propose a two-phase algorithm. In the first phase, we find a minimum risk hazmat tree by solving a binary programming problem. In the second phase, we extend the hazmat tree by adding links in an iterative fashion. Such additions (usually) increase risk but reduce transport cost. Testing with a modest-sized network suggests that our proposed approach is computationally viable.

It is possible to solve a bi-level optimization problem to find a hazmat network that is not restricted to a tree. However, this approach is limited to small problem instances. We reduce the size of the optimization problem by restricting the solution to a tree in the first phase, which allows us to solve large instances of the design problem. In the second phase we increase the density of the hazmat network, where the density (and hence the amount of freedom provided to the carriers) is a design parameter controlled by the local authority.

The hazmat network design problem is perhaps best posed as a bi-criterion (and bi-level) optimization problem. At the first level, the local authority wishes to minimize both cost and risk, and at the second level, the carriers are interested in minimizing cost. It is easy to extend the two-phase method we describe in this paper to the bi-criterion case using a weighted combination of risk and cost as arc attributes. This approach allows the user to generate a number of solutions by changing the weights attached to risk and cost. However, these solutions may not be efficient for the original bi-criterion/bi-level problem, since the two-phase method we propose is only a heuristic.

The two-phase construction-addition approach can be thought of as a general template for hazmat network design. In this paper, we proposed the use of a new model for phase one, namely the optimum communication Steiner tree, which we named MRHT. It is possible to use other models for the generation of the starting network in phase one. For example, one can use the bi-level model to generate an initial network. (On one hand, it is a more general model than MRHT; on the other hand, it is more difficult to solve.) If one wishes to restrict the starting design to a tree, then an alternate objective is to minimize the maximum risk imposed on any arc of the network, instead of the total risk, which will distribute risks 
more equitably on the hazmat tree. The resulting formulation is only slightly larger than the model for MRHT and is no more difficult to solve.

In this paper, we suggested starting with a hazmat tree and expanding it to build a denser hazmat network. An alternate approach is to start with the full network and close roads with the highest risk contributions. This procedure is similar to one of the procedures proposed by Erkut and Ingolfsson [22] to minimize the maximum risk for a single hazmat shipment. It may be worth comparing the addition and deletion approaches computationally in terms of solution quality.

We finish the paper with a caution. The solution to the problem depends on the input data, in particular, the origin-destination flows. Hence, the data must represent long-term shipment patterns as opposed to shipments over a short period of time. If these patterns were to change (for example, due to the installation of a new chemical production facility in the city), then decision makers may have to reconsider their hazmat road network design.

\section{Acknowledgements}

This research was supported by research Grant 25481 from Natural Sciences and Engineering Research Council of Canada. We are thankful to Sarah Bonvicini Mazetti for providing the data for our computational testing.

\section{References}

[1] US Department of Transportation, Hazardous materials shipments. Washington, DC: Research and Special Programs Administration; 1998.

[2] Bureau of Transportation Statistics. Hazardous materials incident reporting system. Washington, DC; 1998.

[3] Government of Alberta. Dangerous goods transportation and handling act, accessed at http://www.qp.gov.ab.ca/ documents/acts/D04.cfm on January 29, 2004; 2002.

[4] Erkut E, Verter V. Modeling of transport risk for hazardous materials. Operations Research 1998;46(5):625-42.

[5] IRR, In: Saccomanno FF, Cassidy K, editors. Transportation of dangerous goods: assessing the risks. Ontario, Canada N2L 3G1: Institute for Risk Research, University of Waterloo; 1993.

[6] CCPS. Guidelines for chemical transportation risk analysis. New York, NY: Center for Chemical Process Safety of the American Institute of Chemical Engineers; 1995.

[7] Erkut E, Verter V, Tjandra S. Hazardous materials transportation. In: Laporte G, Barnhart C, editors. Handbooks in operations research and management science: Transportation. Amsterdam: North-Holland; 2005.

[8] Magnanti TL, Wong RT. Network design and transportation planning: models and algorithms. Transportation Science 1984;18:1-55.

[9] Yang H, Bell MGH. Models and algorithms for road network design: a review and some new developments. Transport Reviews 1998;18:257-78.

[10] Hu TC. Optimum communication spanning trees. SIAM Journal on Computing 1974;3(3):188-95.

[11] Fischetti M, Lancia G, Serafini P. Exact algorithms for minimum routing cost trees. Networks 2002;39(3):161-73.

[12] Ahuja RK, Murty VVS. Exact and heuristic algorithms for the optimum communication spanning tree problem. Transportation Science 1987;21(3):163-70.

[13] Palmer CC, Kershenbaum A. An approach to a problem in network design using genetic algorithms. Networks 1995;26: $151-63$.

[14] Li Y, Bouchebaba Y. A new genetic algorithm for the optimal communication spanning tree problem. Artificial Evolution—Lecture Notes in Computer Science 2000;1829:162-73.

[15] Wu BY, Chao KM, Tang CY. Approximation algorithms for some optimum communication spanning tree problems. Discrete Applied Mathematics 2000;102:245-66. 
[16] Peleg D, Reshef E. Deterministic polylog approximation for minimum communication spanning trees. In: Larsen KG, Skyum S, Winskel G, editors. Lecture notes in computer science: automata, languages and programming, 25th International Colloquium, ICALP'98, vol. 1443. Berlin: Springer; 1998. p. 670-81.

[17] Kara BY, Verter V. Designing a road network for hazardous materials transportation. Transportation Science 2004;38(2): 188-96.

[18] Erkut E, Alp O. A comparison of heuristics for the hazmat network design problem. Research Report 2004-1, Department of Finance and Management Science, University of Alberta School of Business, Edmonton, Alberta, Canada T6G 2R6; 2004.

[19] Garey MR, Johnson DS. Computers and intractability: a guide to the theory of Np-completeness. San Francisco: W.H. Freeman \& Co; 1979.

[20] Gendreau M, Larochelle J-F, Sanso B. A tabu search heuristic for the Steiner tree problem. Networks 1999;34:162-72.

[21] Polzin T, Daneshmand SV. Improved algorithms for the Steiner problem in networks. Discrete Applied Mathematics 2001;112:263-300.

[22] Erkut E, Ingolfsson A. Catastrophe avoidance models for hazardous materials route planning. Transportation Science 2000;34(2):165-79. 